\title{
MITIGATION OF ATMOSPHERIC PHASE DELAY IN INSAR TIME SERIES USING ERA-INTERIM MODEL, GPS AND MODIS DATA: APPLICATION TO THE PERMAFROST DEFORMATION IN HURD PENINSULA, ANTARCTICA
}

\author{
A. R. Reis (1), J. Catalão (1), G. Vieira (2), G. Nico (3) \\ (1) Instituto Dom Luiz (IDL), University of Lisbon, Portugal \\ (2) CEG/IGOT, University of Lisbon, Portugal \\ (3) Consiglio Nazionale delle Ricerche (CNR), Istituto per le Applicazioni del Calcolo, Italy
}

\begin{abstract}
In this study we compare the results obtained using three different atmospheric datasets for the mitigation of atmospheric effects in TerraSAR-X imagery. The used datasets are: ERA-interim re-analysis model, MODIS sensor precipitation water vapour data and GPS derived precipitable water vapour (PWV). The PWV maps were converted to atmospheric path delay and projected into the SAR interferograms geometry. Subsequently the PWV contribution was removed from the interferograms. The Persistent Scatterers technique was applied to the atmospherically corrected interferograms and the obtained displacement rate compared with GPS surface displacement. It was observed that the mitigation of atmospheric effects influences the estimated displacement rate.
\end{abstract}

Index Terms - SAR Interferometry (InSAR), ERA-Interim, GPS, MODIS, Atmospheric phase delay

\section{INTRODUCTION}

Active rock glaciers are indicators of permafrost and their surface kinematics is related to local factors as debris thickness and structure, lithology, topographic relief, ground thermal regime, hydrology, regional climate conditions and can be related to changes in regional or local conditions.

In the last three decades remote sensing methods have been used to measure rock glacier surface deformation [1]. In particular, the synthetic aperture radar interferometry (InSAR) technique, with its unique characteristics to operate day and night and in all weather conditions, is suitable to measure surface deformation in remote regions as Antarctica. However this technique is influenced by several factors that greatly affect the quality of the measurement, such as, the low accuracy of available digital terrain models, reduced temporal window to acquire the scenes (only in the summer months to avoid the decorrelation due to snow accumulation) and the atmospheric path delay. The atmospheric path delay consists of a hydrostatic component stable in time, and a wet component, mainly related to water vapour in the atmosphere, with a large spatial and temporal variability. These variations are difficult to quantify and to model, becoming one of the main factors affecting InSAR applications [2]. Consequently, there is a need to effectively estimate the atmospheric path delay and remove it from interferograms. Existing techniques, such as the persistent scatterers, commonly used to mitigate atmospheric path delay effects in SAR time-series, are not applicable to reduced datasets as those acquired in Antarctica (circa 8 scenes per year).

In this study we compare the results obtained by mitigating atmospheric effects in TerraSAR-X interferograms using three different estimates of phase delay. We use the total column water vapour analysis data ERA-interim, of the European Centre for Medium-range Weather Forecasts (ECMWF), the estimated integrated water vapour data from MODIS measurements and the estimated total zenith path delay of the GPS data. The used data and methods will be presented in section 2 . The results of the comparison between the different approaches for the mitigation of atmospheric path delay and their validation with DGPS observations are described in section 3.

\section{DATA AND METHODS}

We have focused our analysis on Hurd rock glacier in Livingston Island, Antarctica [3], located in the south of Hurd Peninsula (fig. 1). The rock glacier body is $630 \mathrm{~m}$ long and $290 \mathrm{~m}$ wide and the surface shows frequent pressure ridges and furrows, especially in the lower sector. The rock glacier front is $15-20 \mathrm{~m}$ high and shows a slope of 45 degrees [3].

\subsection{GPS Survey}

The surface displacements of this rock glacier have been annually monitored since 2011, using GPS instruments. The measurements have been conducted in late January to early February depending on logistical possibilities. The GPS base station is installed at a reference point in bedrock within the catchment. Measurements have been made in Real Time Kinematics mode by staying for 30 s at each point 
with the rover antenna. The last survey was in Feb 2015 allowing to derive the displacement rate for the last 5 years. (a)

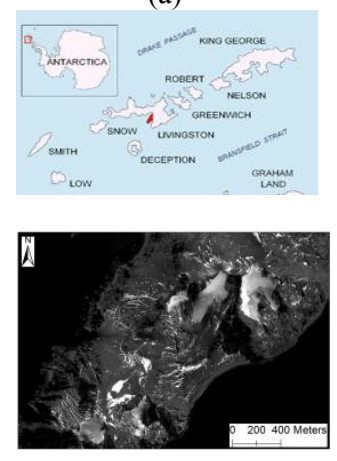

(b)

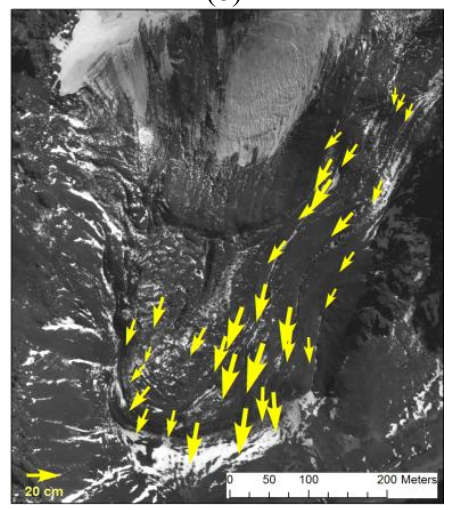

Fig. 1. a) Location of Hurd rock glacier in the Hurd Peninsula, Livingston Island, Antarctica and b) surface displacement measured with D-GPS (horizontal component, $\mathrm{cm} / \mathrm{yr}$ ).

\subsection{Atmospheric mitigation}

The zenith tropospheric delay (ZTD) can be separated into the wet (ZWD) and the hydrostatic (ZHD) components. The hydrostatic component represents about $90 \%$ of the total tropospheric delay, remains stable throughout time and can be accurately estimated using surface temperature and pressure measurements. Contrary, the wet component is highly variable and hard to model. As we are dealing with interferograms, computed as the complex conjugate of two images acquired in two different dates, we are assuming that the ZHD is the same or almost the same in both dates. This means that the ZTD difference between the two dates can be substituted by the ZWD difference. The wet delay is proportional to the atmospheric water vapour content. The relation between PWV and ZWD was given by Bevis [4]:

$$
\mathrm{PWV}=\Pi . \mathrm{ZWD}
$$

The proportional factor $\Pi$ is a function of moist air constants and a variable term defined by the integrated weighted mean temperature. We use this relation, with a $\Pi=0.16$, to convert the ERA-interim PWV and MODIS PWV into the ZWD.

\subsubsection{ERA-Interim model}

The ERA-Interim model is a reanalysis atmospheric global model produced by European Centre for Medium-Range Weather Forecasts (ECMWF). This model offers the Total Column Water Vapor (TCWV) forecast and analysis data every 6 hours. We used Total Column Water Vapor (TCWV) analysis data for the 00:00 and 06:00 UTC with a resolution of 0.125 degrees. The TCWV image closer in time with the SAR acquisition hour was chosen. TCWV was converted to precipitable water vapor and further converted to the zenith wet delay [2].

\subsubsection{Atmospheric GPS data}

We planned to use the GPS permanent station installed at the Spanish station Juan Carlos I circa $2 \mathrm{~km}$ from the study site. But unfortunately the station had data logging gaps and we were forced to use a farther distant GPS station, located at $140 \mathrm{~km}$, the OHI3 station in Antarctic Peninsula. We downloaded the processed Zenith Tropospheric Path Delay (ZTD) data from the International GNSS Service (IGS).

\subsubsection{MODIS data}

The Moderate Resolution Imaging Spectroradiometer (MODIS) is an instrument on board the Terra (EOS AM-1) and Aqua (EOS PM-1) satellites. Terra (descending mode) and Aqua (ascending mode) MODIS are viewing the entire Earth's surface every 1 to 2 days. We used the MODIS Precipitable Water Vapor, Level 2 Products (MOD2L5), from TERRA MODIS and AQUA MODIS instruments with a resolution of $1 \times 1 \mathrm{~km}$ using the Near-IR channel. This product includes information about geolocation data and time, cloud mask and quality assurance. The MODIS images were selected by analyzing the values indicated in the Cloud mask and quality assurance. The PWV MODIS measurements however are sensitive to the presence of clouds, which limits significantly its use in cloudy regions because only PWV values collected under clear sky conditions have quality to be used. The selection of images was based on the following criterion: a) image acquisition time closer to the time of acquisition of SAR image; b) image with quality (given by the quality assurance) and c) image without clouds (given by Cloud Mask). Systematic biases in PWV MODIS measurements may exist and need to be calibrated with more accurate PWV measurements (e.g. GPS PWV) [5]. In this case, the data from OHI3 IGS station (section 2.3) was used. The MODIS PWV image was then resampled to the INSAR geometry and converted to ZTD using the relation (1).

\subsection{InSAR data}

A set of 15 images from TerraSAR-X satellite in ascending and descending mode were used in this study. The images were acquired for the Antarctic summer between January and March 2014. The resulting time series of perpendicular and temporal baselines are shown on Table 1. The images were interferometrically processed in order to have a master date minimizing the temporal baseline, resulting in 6 and 7 interferograms for the descending and ascending pass respectively. The DORIS software (Delft University of Technology) was used for interferometric processing and to correct the topographic effects of Earth's curvature a digital terrain model was used. Since, a high degree of temporal decorrelation was verified, we decided to use the persistent scatterers technique to estimate the displacement rate and the height error using the STAMPS software [6]. 
Table 1. Perpendicular and temporal baselines.

\begin{tabular}{|c|c|c|c|}
\hline \multicolumn{2}{|c|}{ Ascending orbit } & \multicolumn{2}{|c|}{ Descending orbit } \\
\hline $\begin{array}{c}\text { Date of } \\
\text { acquisition }\end{array}$ & $\begin{array}{c}\text { Baseline } \\
\text { (m) }\end{array}$ & $\begin{array}{c}\text { Date of } \\
\text { acquisition }\end{array}$ & $\begin{array}{l}\text { Baseline } \\
\text { (m) }\end{array}$ \\
\hline 2-jan-2014 & -189 & 3-jan-2014 & -169 \\
\hline 13-jan-2014 & -196 & 14-jan-2014 & -144 \\
\hline 24-jan-2014 & -70 & 25-jan-2014 & 40 \\
\hline 04-feb-2014 & 0 & 5-feb-2014 & 0 \\
\hline 15 -feb-2014 & -69 & 16-feb-2014 & -109 \\
\hline 26-feb-2014 & -75 & $27-\mathrm{feb}-2014$ & -66 \\
\hline 9-mar-2014 & -194 & 21-mar-2014 & 56 \\
\hline 20-mar-2014 & -48 & & \\
\hline
\end{tabular}

The previously estimated precipitable water vapour from ERA-Interim and MODIS were converted into zenith wet delay using relation (1). The zenith wet delay is then projected into the satellite line of sight using the incidence angle (23 degree) and resampled to the interferogram spatial resolution $(3 \mathrm{~m})$. The resulting atmospheric phase screen is removed from the interferograms and the persistent scatterers technique applied to the 6 stacks of interferograms (ascending/descending plus ERA, MODIS, GPS).

\section{RESULTS AND CONCLUSIONS}

In this section we summarize some results obtained by applying the proposed methodology to generate surface deformation maps. Figure 2 (a-d) show the PS estimated displacement rate using the ascending SAR images. In Hurd rock glacier, the number of PS's is almost identical with a similar spatial distribution covering most of the rock glacier front. Concerning the displacement rate for each solution, it was found that there is an overall good agreement in the spatial distribution pattern and on the velocity amplitude. It seems that the atmospheric mitigation does not affect the estimated PS velocity. However, in other areas of the island, considerable differences in the displacement rate are observed between the original solution and the atmospheric free solutions, figure 2e) and f), respectively. In this area, the effect of the atmospheric delay mitigation is clear, contributing to a more homogeneous solution and to a different displacement rate value.

For some selected PS close to the GPS marks, the displacement time series along a three month period was plotted. In figures $2 \mathrm{~g}$ ) and $\mathrm{h}$ ) the descending pass original and MODIS solution, are shown and in figures $2 \mathrm{j}$ ) and $\mathrm{m}$ ) the corresponding solutions for the ascending pass. We observe that the atmospheric mitigation has a strong impact on the time series revealing second order effects on the displacement not seen in the original solution. These effects may improve our knowledge about the thawing process and can be correlated with temperature or snow cover variations during the summer. The change in the sign of the trend is related with displacement mechanisms that can be dominated by vertical subsidence (increase in the LOS) or by horizontal displacement (in this case decrease in LOS for the ascending pass). A good example is shown in Figure $2 \mathrm{~m})$.

The estimated LOS velocities were also compared with the GPS survey. For that the GPS 3D velocities were projected onto the line of sight for the ascending and desceding pass. In figure 2i) and $n$ ) the GPS LOS and the PS's displacements vs time are shown. The absolute reference for PS is given by the 2013 GPS survey. The trend was estimated using only the PS' displacement. GPS trend and InSAR trend are diferent due to diferent sampling resolution in time.

The main conclusions are: a) the mitigation of atmospheric effects influences the estimated displacement rate; b) the mitigation of atmospheric artifacts reduces the spatial dispersion of velocity estimates, c) on Hurd rock glacier the effect of atmospheric mitigation is reduced. While there is a significant change in the time series profiles, this effect is particularly evident in the approach with MODIS data, d) major influence of the atmospheric mitigation is seen on the displacement time series.

\section{AKNOWLEDGDEMENTS}

This work is part of project PERMANTAR-3 (PTDC/AAGGLO/3908/2012) - Permafrost and Climate Change in the Antarctic Peninsula, funded by the FCT. TerraSAR-X imagery has been obtained through the project DLR LAN1276. Logistics was provided by the Portuguese Polar Program, the Bulgarian Antarctic Institute and the Spanish Antarctic Program. Personnel from the stations St Kliment Ohridski and Juan Carlos I are thanked for the field support.

\section{REFERENCES}

[1] A. Kaab, "Remote Sensing of Permafrost-related Problems and hazards". Permafrost and Periglacial Processes, 19, 107-136, doi:10.1002/ppp.619, March 2008.

[2] G. Nico, R. Tomé, J. Catalão, P.M.A. Miranda,"On the use of the WRF model to mitigate tropospheric phase delay effects in SAR interferograms". IEEE Transactions on Geoscience and Remote Sensing, Vol. 49, Issue 12, 4970 - 4976, doi:10.1109/TGRS.2011.2157511, December 2011.

[3] C. Hauck, G. Vieira, S. Gruber, J. Blanco, M. Ramos, "Geophysical identification of permafrost in Livingston Island, Maritime Antarctic". Journal of Geophysical Research, VOL. 112, F02S19, doi: 10.1029/2006JF000544, June 2007.

[4] M. Bevis, S. Businger, T. A. Herring, C. Rocken, R. A. Anthes, R. H. Ware, "GPS Meteorology: Remote Sensing of Atmospheric Water vapour Using the Global Positioning System". Journal of Geophysical Research, VOL 97, NO. D14, PAGES 15, 787-15, 801, October 1992.

[5] P. Mateus, G. Nico, J. Catalão, "Can spaceborn SAR interferometry be used to study the temporal evolution of PWV?" Atmospheric Research, 119, 70-80, doi:10.1016/j.atmosres.2011.10.002, October 2011.

[6] A. Hooper, P. Segall, and H. Zebker, "Persistent scatterer interferometric synthetic aperture radar for crustal displacement analysis, with application to Volcano Alcedo, Galapagos," J. Geophysical. Research-Solid Earth, vol. 112, no. B7, p. B07407, 2007. 
(a)

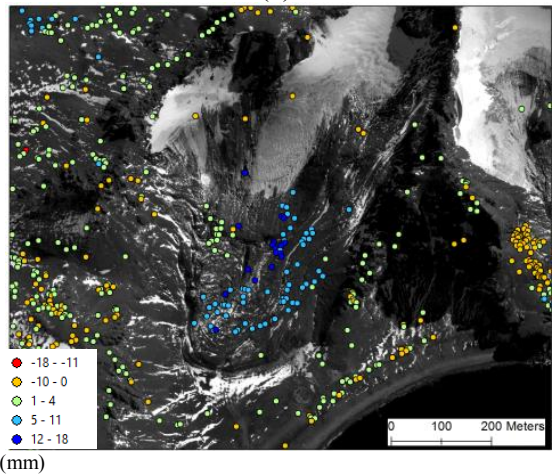

(d)

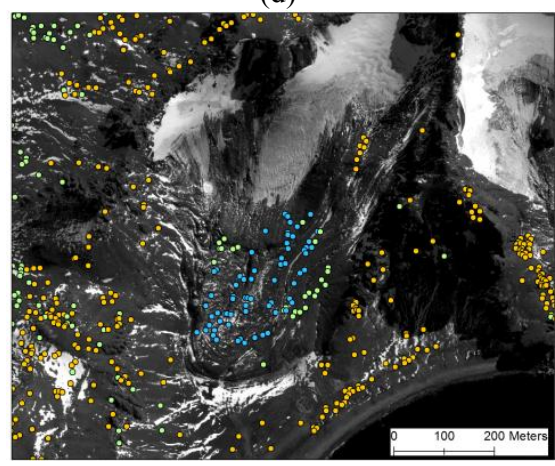

(g)

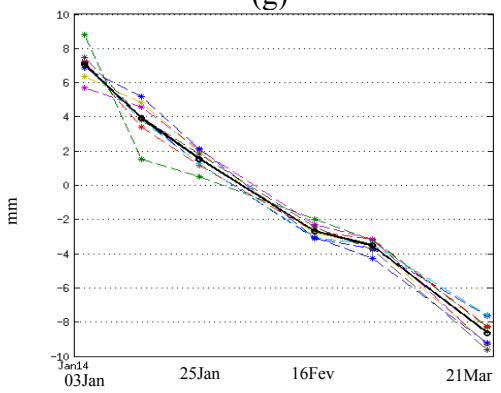

(j)

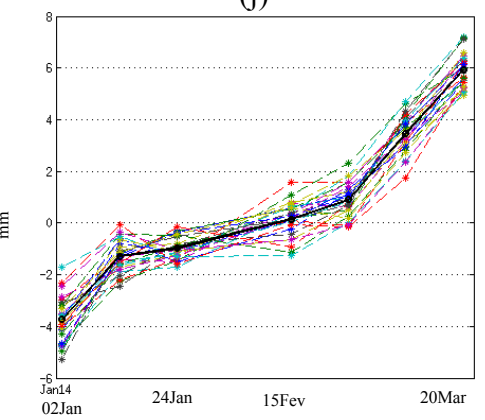

(b)

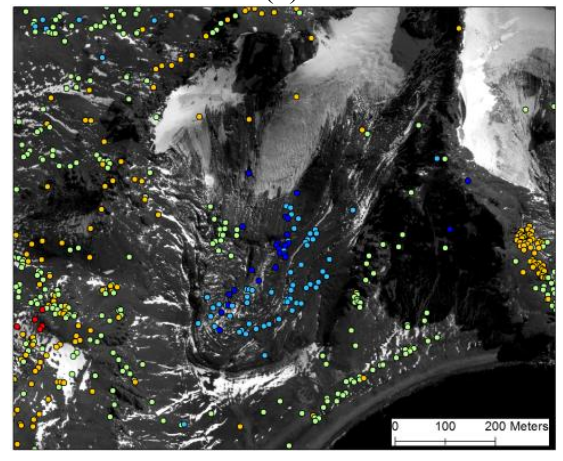

(e)

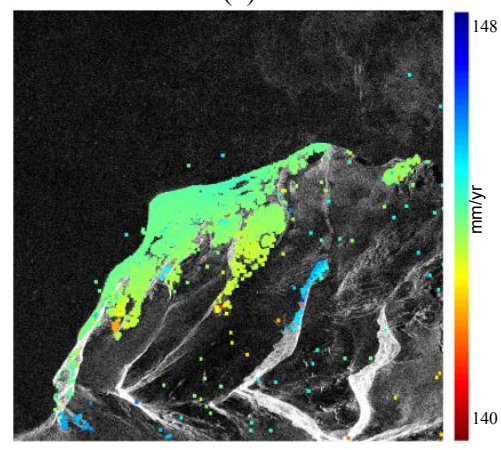

(h)

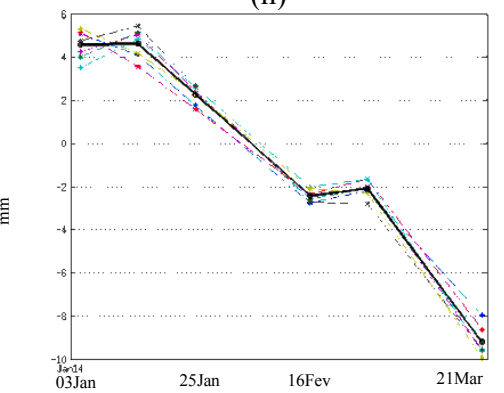

(m)

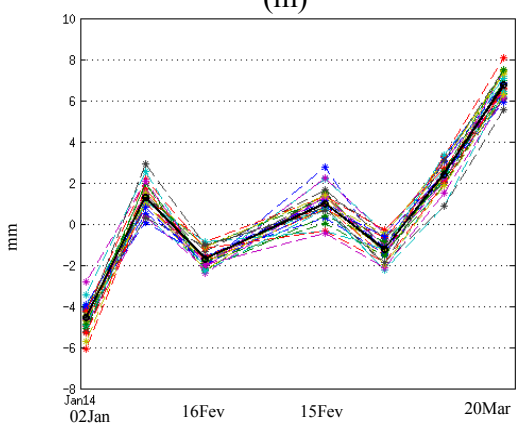

(c)

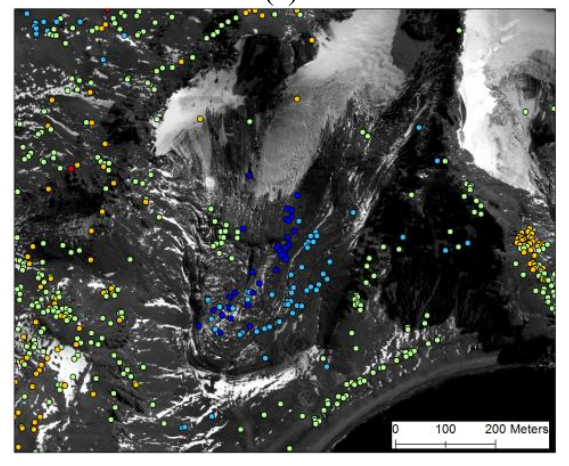

(f)

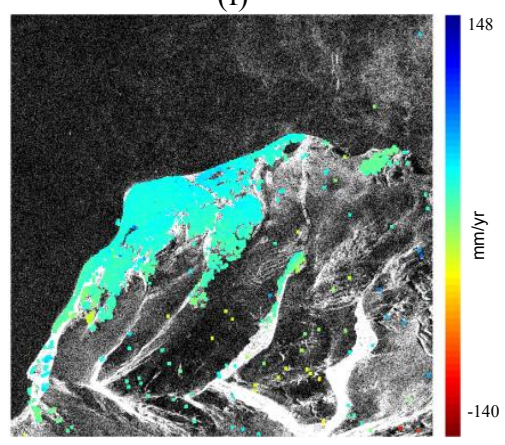

(i)

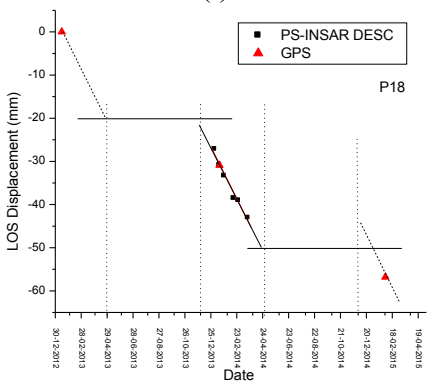

(n)

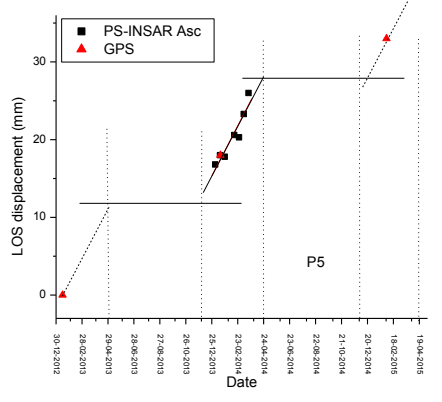

Figure 2. a) PS displacement, no atmospheric effects mitigation; b) PS displacement, ERA-Interim data; c) PS displacement, GPS atmospheric data; d) PS displacement, MODIS data; e) Mac Kay rock glacier PS displacement; no atmospheric mitigation; f) Mac Kay rock glacier PS displacement atmospheric mitigation with MODIS data; g) PS displacement time series, descending pass; h) PS displacement time series, atmospheric mitigation with MODIS data; i) comparison with GPS survey, descending pass; j) PS displacement time series, ascending pass; m) PS displacement time series, atmospheric mitigation with MODIS data; $\mathrm{n}$ ) comparison with GPS survey, ascending pass. 